\title{
A VANISHING THEOREM FOR COHOMOLOGY
}

\author{
J. L. ALPERIN ${ }^{1}$ AND DANIEL GORENSTEIN ${ }^{2}$
}

Abstract. A criterion is given for $H^{0}(G, A)=H^{1}(G, A)=0$, where $G$ is a group and $A$ is a $G$-module, in terms of the cohomology of a collection of subgroups of $G$.

In computing the cohomology of a group, knowledge of the cohomology of the subgroups is often useful. The following result is of that nature:

THEOREM. Let $\mathscr{L}$ be a collection of subgroups of the group $G$ and let $A$ be a G-module. Assume that $\mathscr{L}$ has a minimum element and that $G$ is generated by the subgroups in $\mathscr{L}$. It follows that if, for each $L$ in $\mathscr{L}$,

then

$$
H^{0}(L, A)=H^{1}(L, A)=0
$$

$$
H^{0}(G, A)=H^{1}(G, A)=0 .
$$

Proof. If $L_{0}$ is the minimum element of $\mathscr{L}$, then $L_{0}$ has no fixed points on $A$, as $H^{0}\left(L_{0}, A\right)=0$, so neither does $G$; hence $H^{0}(G, A)=0$. To show that $H^{1}(G, A)=0$ it suffices to prove that if

$$
0 \rightarrow A \rightarrow E \rightarrow Z \rightarrow 0
$$

is an exact sequence of $G$-modules, with $Z$ the integers with trivial action, then the extension splits; this is because $H^{1}(G, A) \simeq \operatorname{Ext}_{Z G}^{1}(Z, A)$.

However, if $L \in \mathscr{L}$, then $H^{1}(L, A)=0$; so $E=A \oplus Z_{L}$, where $Z_{L}$ is an $L$ module isomorphic to $Z$. Moreover, since $H^{0}(L, A)=0$, we have that $Z_{L}=E^{L}$, the fixed points of $L$ in $E$. Since $L_{0} \subseteq L$ we have $Z_{L} \subseteq Z_{L_{0}}$ so $Z_{L}=Z_{L_{0}}$ for every $L$ in $\mathscr{L}$. But $G$ is generated by all the subgroups $L$, so $Z_{L_{0}}$ is also $G$-invariant and $E$ splits over $A$ as a $G$-module.

We conclude with an example which at the same time illustrates the use of this result and limits possible generalizations. Let $G=\mathrm{GL}(4,2)$ and let

Received by the editors April 27, 1971.

AMS 1970 subject classifications. Primary $20 \mathrm{~J} 05$.

Key words and phrases. Cohomology groups, general linear groups, ext functor.

${ }^{1}$ Supported in part by National Science Foundation Grant NSF GP-28700.

${ }^{2}$ Supported in part by National Science Foundation Grant NSF GP-16640. 
$A$ be a vector space of dimension four over GF(2) with $G$ acting in the usual way. Let

$$
S=\left(\begin{array}{llll}
0 & 1 & 0 & 0 \\
1 & 1 & 0 & 0 \\
0 & 0 & 1 & 0 \\
0 & 0 & 0 & 1
\end{array}\right), \quad T=\left(\begin{array}{llll}
1 & 0 & 0 & 0 \\
0 & 1 & 0 & 0 \\
0 & 0 & 0 & 1 \\
0 & 0 & 1 & 1
\end{array}\right)
$$

and set $L_{0}=\langle S, T\rangle, L_{1}=C_{G}(S T)$ and $L_{2}=C_{G}\left(S T^{-1}\right)$. It is easily verified, as $L_{j} \simeq \mathrm{GL}(2,4), j=1,2$, and $\operatorname{GL}(2,4)$ has a center of odd order, that

$$
H^{i}\left(L_{k}, A\right)=0, \quad i \geqq 0, k=0,1,2 .
$$

Moreover, $L_{1} \supseteq L_{0}, L_{2} \supseteq L_{0}$ and $G=\left\langle L_{0}, L_{1}, L_{2}\right\rangle$. Hence, by our theorem $H^{0}(G, A)=H^{1}(G, A)=0$, a very special case of a theorem of D. G. Higman [2]. However, $H^{2}(G, A) \neq 0$ as Blackburn [1] has proved.

\section{REFERENCES}

1. N. Blackburn, The extension theory of the symmetric and alternating groups, Math. Z. 117 (1970), 191-206.

2. D. G. Higman, Flag-transitive collineation groups of finite projective spaces, Illinois J. Math. 6 (1962), 434-446. MR 26 \#663.

Department of Mathematics, University of Chicago, Chicago, Illinois 60637

Department of Mathematics, Rutgers University, New Brunswick, New JERSEY 08903 